\title{
A Multicenter Survey of Acute Stroke Imaging Protocols for Endovascular Thrombectomy
}

\author{
Byungjun Kim, $\mathrm{MD}, \mathrm{PhD}^{1}$, Sung-Hye You, $\mathrm{MD}, \mathrm{PhD}^{1}$, Seung Chai Jung, $\mathrm{MD}, \mathrm{PhD}^{2}$ \\ ${ }^{1}$ Department of Radiology, Anam Hospital, Korea University College of Medicine, Seoul, Korea \\ ${ }^{2}$ Department of Radiology and Research Institute of Radiology, Asan Medical Center, University of Ulsan College of Medicine, Seoul, Korea
}

\begin{abstract}
Purpose: Identifying current practices in acute stroke imaging is essential for establishing optimal imaging protocols. We surveyed and assessed the current status of acute stroke imaging for endovascular thrombectomy (EVT) at tertiary hospitals throughout South Korea.
\end{abstract}

Materials and Methods: An electronic questionnaire on imaging protocols for EVT in patients with acute ischemic stroke was e-mailed to physicians at 42 registered tertiary hospitals, and their responses were collected between February and March 2020.

Results: Of the 36 hospitals participating in the survey, 69\% (25/36) adopted computed tomography (CT)-based protocols, whereas 31\% (11/36) adopted magnetic resonance (MR)-based protocols. Non-enhanced CT (NECT) was the initial imaging study at 28\%, NECT with CT angiography (CTA) at 36\%, and NECT with CTA and CT perfusion (CTP) at 33\% of hospitals. Perfusion imaging was performed at 61\% (22/36), CTP at 44\% (16/36), and MR perfusion at 17\% (6/36) of hospitals. Multiphase CTA was performed at $67 \%$, single-phase CTA at 11\%, time-of-flight MR angiography (MRA) at $8 \%$, contrast-enhanced MRA at $8 \%$, and both at $6 \%$ of hospitals. For late time window stroke, $50 \%$ of hospitals used identical imaging protocols to those for early time window stroke, 39\% used additional MR imaging (MRI), and 6\% converted the imaging strategy from CT to MRI. Post-processing programs were used at 28\% (10/36), and RAPID software at $14 \%(5 / 36)$ of hospitals, respectively. Most hospitals (92\%) used the same imaging protocols for posterior and anterior circulation strokes.

Conclusion: Our multicenter survey demonstrated considerable heterogeneity in acute stroke imaging protocols across South Korean tertiary hospitals, suggesting that hospitals refine their imaging protocols according to hospital-specific conditions.

Key Words: Acute stroke; Thrombectomy; Cerebrovascular stroke

\section{INTRODUCTION}

Previous clinical trials have demonstrated the safety and efficacy of endovascular thrombectomy (EVT) in patients with acute ischemic stroke (AIS) caused by intracranial large vessel occlusion (LVO); therefore, workflow optimization emerged as an important factor to reproduce successful trial results in real-world clinical practice. ${ }^{1-3}$ An optimal workflow is mainly founded on an optimal imaging strategy for implementing timely thrombectomy in patients who are likely to benefit from EVT. ${ }^{4}$ Therefore, the imaging strategy should be organized efficiently and well-integrated into the workflow.

\section{Correspondence to: Byungjun Kim, MD, PhD}

Department of Radiology, Anam Hospital, Korea University College of Medicine, 73 Goryeodae-ro, Seongbuk-gu, Seoul 02841, Korea

Tel: +82-2-920-5693

Fax: +82-2-929-3796

E-mail: cardillo@hanmail.net

Received: May 27, 2020

Revised: October 16, 2020

Accepted: October 27, 2020
Copyright $\odot 2021$ Korean Society of Interventional Neuroradiology

This is an Open Access article distributed under the terms of the Creative Commons Attribution Non-Commercial License (http://creativecommons.org/licenses/by-nc/4.0) which permits unrestricted non-commercial use, distribution, and reproduction in any medium, provided the original work is properly cited.

pISSN 2093-9043 eISSN 2233-6273 
Although the imaging strategies introduced in a series of positive trials were computed tomography (CT)-based protocols, the detailed imaging selection criteria differed to a certain degree. ${ }^{5-9}$ Recently, 2 endovascular trials have demonstrated the clinical benefits of EVT in a late time window using perfusion-weighted imaging in conjunction with post-processing software. ${ }^{10,11}$ Establishing a broadly accepted consensus for an optimal imaging protocol for patients with AIS due to LVO is currently difficult. In this study, we sought to identify a broad range of imaging protocols for EVT in hospital workflows by conducting a multicenter survey. Furthermore, we aimed to provide opportunities for refining the imaging strategies by identifying various solutions adopted at different hospitals and suggesting areas where consensus on universal best practices is needed in future clinical trials.

\section{MATERIALS AND METHODS}

The electronic survey was conducted over 2 months between February and March 2020. The target hospitals were tertiary hospitals designated as local or regional emergency medical centers in South Korea and endovascular treatment-capable institutions certified by the Korean Society of Interventional Neuroradiology (KSIN). Contact information for the hospitals was obtained from the KSIN website, and an attempt was made to contact the representative neurointerventionist or neuroradiologist at each hospital via telephone or e-mail.

Hospitals that expressed interest in participating in the survey were e-mailed a questionnaire in Korean. The questionnaire included 12-13 multiple-choice questions about imaging protocols for EVT in patients with AIS due to LVO (Supplementary Material). The questions were categorized into imaging protocols in an early time window $(<6$ or 8 hours after symptom onset), those in a late time window (between 6 or 8 hours and 24 hours), and those in patients with LVO in the posterior circulation. Responses were stored in an electronic database and analyzed using standard descriptive statistics (Microsoft Excel; Microsoft, Redmond, WA, USA). Financial compensation was offered to the study participants.

\section{RESULTS}

The South Korean government has designated 150 hospitals as local or regional emergency medical centers. Among them, 108 hospitals did not have any members registered in the KSIN; hence, they were excluded. We attempted to contact the neurointervention team members at the remaining 42 hospitals while considering their regional arrangement.

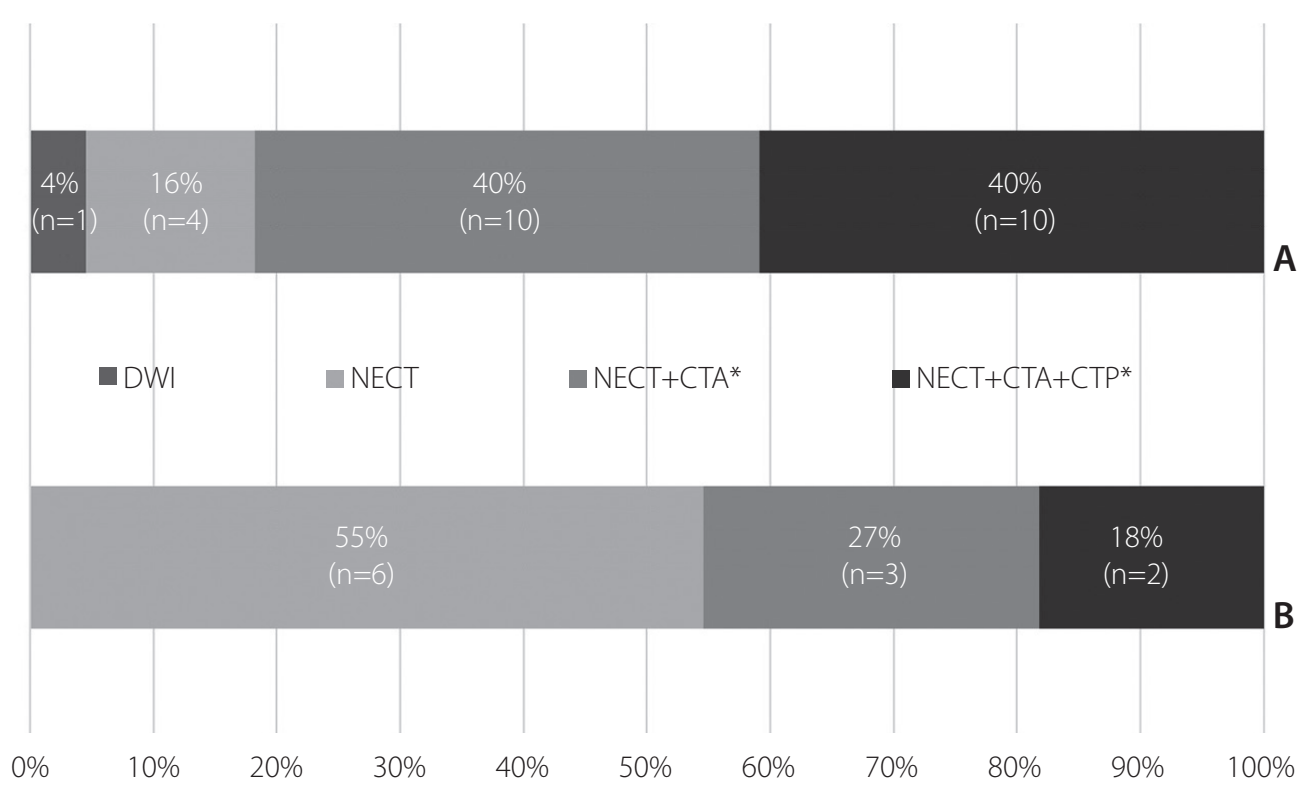

Fig. 1. Summary of initial imaging modalities adopted at the participating hospitals. (A) Hospitals adopting computed tomography (CT)-based imaging protocols. (B) Hospitals adopting magnetic resonance-based imaging protocols. DWI, diffusion-weighted imaging; NECT, non-enhanced CT; CTA, CT angiography; CTP, CT perfusion. *"+" implies imaging acquisition in a single session. 
Of these hospitals, 86\% (36/42) completed the survey. These included 19 (53\%) regional and 17 (47\%) local emergency medical centers.

\section{Imaging protocols in an early time window}

Among the participating hospitals, 69\% (25/36) adopted CT-based imaging protocols to facilitate triage for EVT in an early time window, whereas 31\% (11/36) adopted magnetic resonance (MR)-based imaging protocols. Four of 16 hospitals (25\%) in Seoul and 7 of 20 hospitals (35\%) in the rest of the country used MRI for acute stroke imaging work-up prior to EVT. In addition, 4 of 19 hospitals (21\%) designated as regional emergency centers and 7 of 17 hospitals (41\%) with local emergency centers reported that they utilize MRI in the setting of AlS.

Non-enhanced CT (NECT) was the preferred initial imaging modality at 97\% (35/36) of hospitals, and diffusion-weighted imaging (DWI) was used in single hospitals (3\%, 1/36). NECT was the sole initial imaging method at $10(28 \%)$, NECT with CT angiography (CTA) at 13 (36\%), and NECT with CTA and CT perfusion (CTP) at 12 (33\%) of hospitals (Fig. 1). At 11 hospitals adopting MR-based protocols, initial CT examinations were followed by further acquisition of MRI.

Perfusion-weighted imaging was incorporated into the routine imaging protocols at $64 \%$ (23/36) of hospitals. CTP was the preferred imaging technique at $44 \%(16 / 36)$ of hospitals. MR perfusion (MRP) was used at 17\% (6/36) of hospitals, and 1 hospital routinely used both CTP and MRP (Fig. 2).

Intravenous thrombolysis using tissue plasminogen activator (tPA) was performed immediately after the initial imaging studies, with NECT being performed at 28\% (10/36), DWI at 3\% (1/36), NECT with CTA at 36\% (13/36), and NECT with CTA and CTP at 30\% (11/36) of hospitals. Vascular imaging is routinely obtained as part of the imaging work-up of AIS patients before intravenous tPA in 31\% (11/36) of sites. Furthermore, single hospitals $(3 \%, 1 / 36)$ answered that they performed IV tPA after acquisition of further MRI data.

For precluding contrast-induced nephrotoxicity, 20\% (5/25) of the hospitals adopting CT-based protocols routinely checked the patients' serum creatinine levels, while 56\% (14/25) checked this only when the patients' history of kidney disease was confirmed. All hospitals adopting MR-based protocols did not routinely or selectively check the glomerular filtration rate before administering MR contrast agents.

At 82\% (21/25) of the hospitals adopting CT-based protocols, multiphase CTA was routinely used for evaluating intracranial LVO and collateral status. Among 4 hospitals using single-phase CTA, 3 routinely obtained CTP data, and the remaining 1 used only NECT and single-phase CTA (Fig. 3).

At $45 \%(5 / 11)$ of the hospitals adopting MR-based proto-
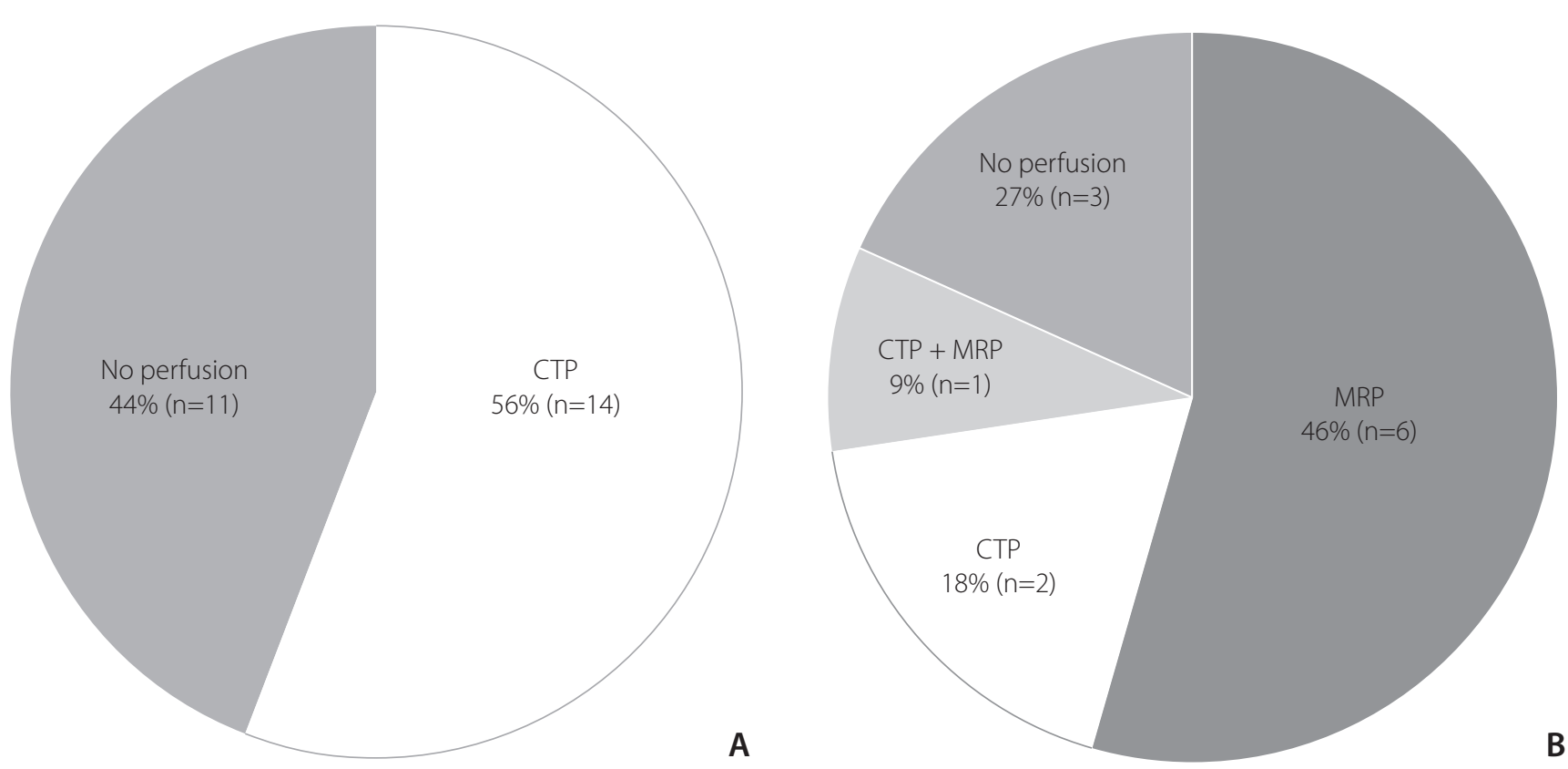

Fig. 2. Use of perfusion-weighted imaging in routine protocols. (A) Hospitals adopting computed tomography (CT)-based imaging protocols. (B) Hospitals adopting magnetic resonance (MR)-based imaging protocols. CTP, CT perfusion; MRP, MR perfusion. 
cols, a fast MRI technique using modified MR parameters was applied to reduce image acquisition time. The others used conventional MR protocols with simplified sequences. Detailed MR sequences included DWI, gradient echo or susceptibility-weighted images, and fluid-attenuated inversion

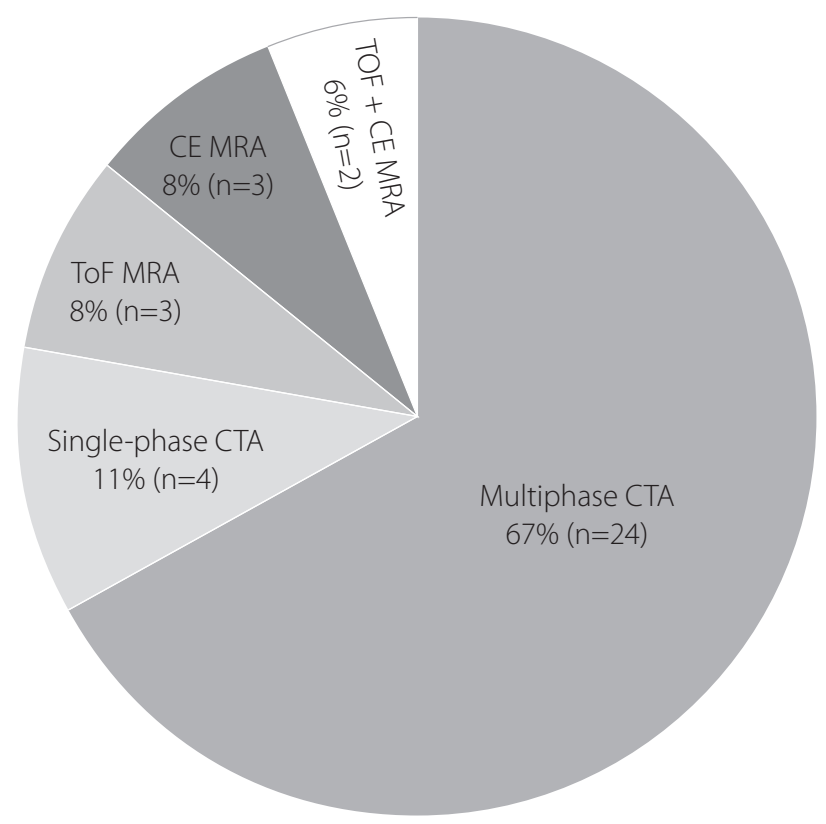

Fig. 3. Detailed technique of noninvasive vascular study in routine protocols. CTA, computed tomography angiography; ToF MRA, timeof-flight magnetic resonance angiography; CE MRA, contrast-enhanced magnetic resonance angiography. recovery (FLAIR) or T2 images. Time-of-flight MR angiography (MRA) was used at 27\% (3/11), contrast-enhanced MRA at $27 \%(3 / 11)$, and both at $18 \%(2 / 11)$ of hospitals. The remaining 3 (27\%) hospitals used multiphase CTA instead of other MRA techniques (Fig. 3).

\section{Imaging protocols in a late time window}

Half of the participating hospitals (50\%, 18/36) assessed patients with AIS using the same imaging protocols in both the early and late time windows. Among the 25 hospitals adopting CT-based protocols, 14 (56\%) performed additional MRI techniques, including DWI, FLAIR, and MRP, and 2 (8\%) converted the imaging strategy from CT-based protocols to MR-based protocols. However, most hospitals adopting MRbased protocols $(82 \%, 9 / 11)$ maintained their imaging strategy regardless of the elapsed time between symptom onset and patient presentation. One hospital acquired additional MRP, and another switched the MRI technique from fast to conventional sequences for enhancing image resolution. The 10 hospitals that used a combination of NECT and CTA alone as the routine imaging protocol used MRI instead of CTP in the late time window (Fig. 4).

Post-processing programs were used at 28\% (10/36) hospitals. RAPID (iSchemaView, Menlo Park, CA, USA) was the most popular software $(50 \%, 5 / 10)$, followed by Olea Sphere (Olea Medical, La Ciotat, France) (20\%, 2/10). Vitrea Intelligence (Vital Images, Minnetonka, MN, USA), JBS (JLK Inspection), and an

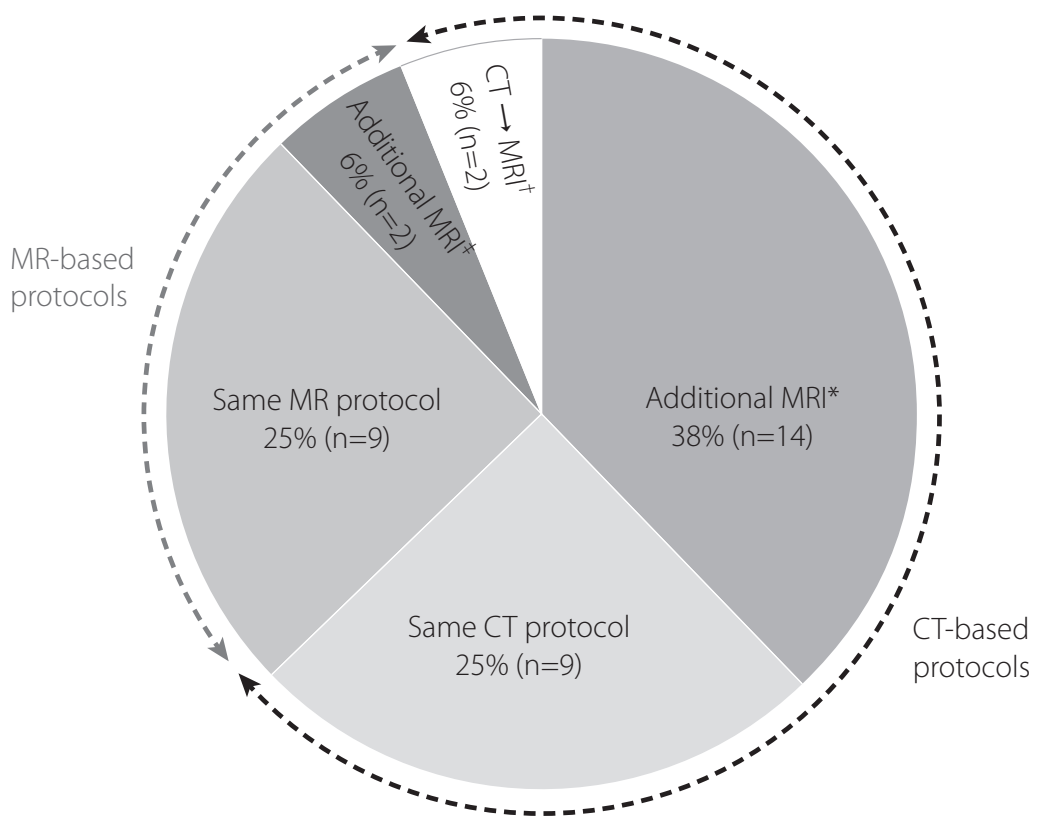

Fig. 4. Routine imaging protocols for patients presenting in a late time window. ${ }^{*}$ Further acquisition of magnetic resonance imaging (MRI) after routine computed tomography (CT)-based imaging protocols used in patients presenting in an early time window. ${ }^{\dagger}$ Converting the routine imaging strategy from CT-based to MR-based protocols. ${ }^{\ddagger}$ Further acquisition of MR perfusion or switching fast MR to conventional MR techniques. 
in-house program were used at the remaining hospitals (30\%, 3/10) (Fig. 5).

\section{Imaging protocols in patients with LVO in the posterior circulation}

For patients with posterior circulation stroke, 75\% (27/36) hospitals performed EVT according to the imaging findings within 24 hours after stroke onset. However, EVT was implemented only if the symptom duration had not exceeded 12 and 10 hours at 7 (19\%) and 2 (6\%) hospitals, respectively.

At 92\% (33/36) of hospitals, patients with posterior circulation stroke were assessed using the same imaging protocols as those with anterior circulation stroke. Among the 25 hospitals adopting CT-based protocols, 3 additionally performed DWI for patients with posterior circulation stroke, even in an early time window. Therefore, the overall frequency of DWI use was 42\% (15/36) and 78\% (28/36) in early and late time windows, respectively.

\section{DISCUSSION}

An optimal imaging protocol should promptly and accurately identify intracranial LVO, small infarct core, large core-perfusion mismatch, and good collaterals, which warrant EVT. ${ }^{12}$ Considering the overall importance of the imaging strategy in establishing an optimal workflow, consensus on an efficient imaging protocol is necessary for each hospital to re-

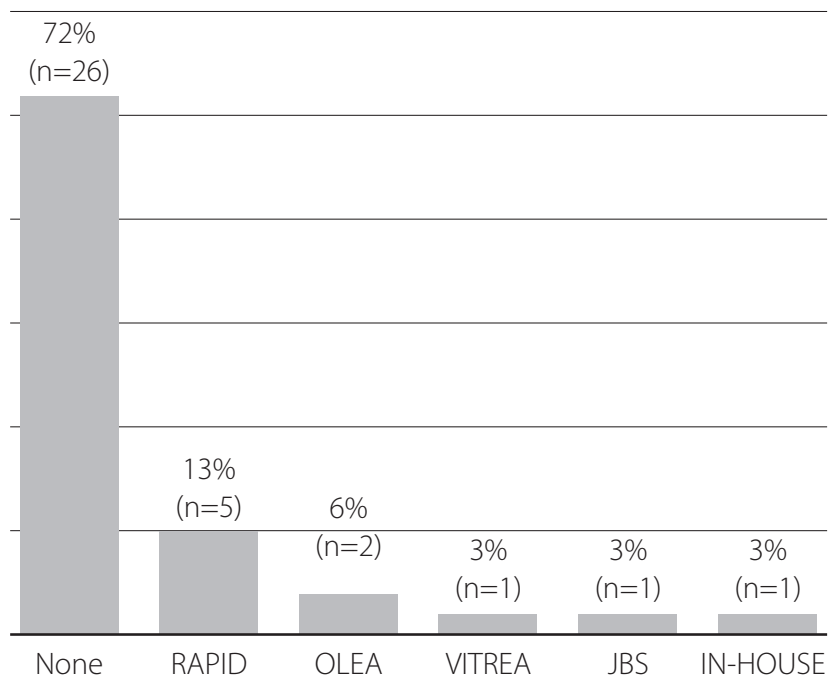

Fig. 5. Current usage status of post-processing software programs. OLEA, olea sphere; VITREA, vitrea intelligence; IN-HOUSE, in-house program. fine the imaging-based selection of patients eligible for EVT. In the present multicenter survey, we identified considerable heterogeneity in imaging protocols between tertiary hospitals in South Korea. As the imaging protocol is inherently influenced by the medical resources available at each hospital, adopting a uniform protocol including the same modality is impossible. Therefore, by reviewing the wide variations in imaging protocols at many hospitals, we tried to provide an opportunity for hospitals to redesign their systems, which might have been influenced by reasons such as the preference of individual physicians and unawareness of effective solutions applied at other hospitals.

\section{Current status of MR usage in actual clinical practice}

The practical challenges for implementing MRI for acute stroke include limited availability during 24 hours a day and 7 days a week, relatively longer scan time than that required for $C T$, need to screen patients with contraindications to $M R I$, and vulnerability to patient movement. Therefore, an efficient process to screen patients for their eligibility to undergo MRI, a prioritization system for patients scheduled to undergo MRI, a physical proximity of the MR scanners to the emergency room, and a sufficient MR scanning capacity to accommodate unscheduled patients at all times are necessary to offer MR-based protocol in the setting of AIS. ${ }^{13}$ Most of the successful endovascular trials that proved the clinical benefits of EVT used MRI in only a minority of their cohort. ${ }^{5-9}$ Reflecting the imaging strategies in those trials, more than half $(69 \%, 25 / 36)$ of the hospitals surveyed used CT-based imaging protocols to select patients for EVT in real-world practice. Moreover, there was no significant difference in routine imaging modality for stroke patients according to geographical regions and between local and regional emergency medical centers. Although MR-based protocol needs medical resources that go beyond having an MR scanner open 24/7, the general trend toward CT can be explained by abundant advantages of $C T$ imaging itself in the acute setting.

In a late time window stroke, we discovered that $75 \%$ (27/36) of hospitals used MRI to select patients eligible for EVT. DWI is the most sensitive and specific imaging technique for detecting an acute infarct, which is crucial in the triage for late time window stroke. ${ }^{14}$ Furthermore, we found that some hospitals $(12 \%, 3 / 25)$ adopting CT-based protocols additionally performed DWI to accurately assess the baseline extent of infarct core when posterior circulation stroke 
was confirmed. A recent multicenter study has revealed that even in cases of early time window stroke, MR-based selection could be accomplished within a timeframe similar to that for CT-based selection without delaying treatment or impacting clinical outcomes. ${ }^{15}$ Therefore, the use of MRI is expected to become widespread with advances in the fast scanning technique and because it offers abundant information necessary for decision making under complex clinical circumstances. However, previous trials have suggested that MRI should be used only if hospitals have secured sufficient triaging efficiency similar to CT-based protocols, and this warrants dedicated training to overcome major obstacles for using MRl at hospitals where it is available., ${ }^{4,15}$

\section{Rapid implementation of CTA and current usage status of multiphase CTA}

Identification of LVO in association with acute stroke is an essential process for determining whether to proceed with EVT. CTA is a rapid and reliable imaging technique when screening patients with AIS due to LVO. The ESCAPE trial has recommended that NECT should be followed by CTA in a single session with a "no turn back approach" to the emergency room following brain imaging. ${ }^{16}$ According to the detailed workflows, intravenous tPA could be prepared after ruling out intracranial hemorrhage on NECT and during further acquisition of $\mathrm{CT}$ and then administered in the $\mathrm{CT}$ unit after CTA. Our results showed that most hospitals adopting CT-based protocols $(20 / 25,80 \%)$ and nearly half of those using MR-based protocols $(5 / 11,45 \%)$ routinely performed NECT and CTA in a single session to expedite decision making.

The rate of infarct growth is contingent upon the robustness of the collateral circulation. ${ }^{17}$ Although assessment of collateral status is not directly included in eligibility criteria for EVT in a late time window stroke, the international stroke expert groups strongly recommend assessing collateral circulation as well as target mismatch for therapeutic decision-making. ${ }^{12}$ In addition, the 2019 update of the Korean clinical practice guidelines has remarked that collateral assessment should be considered for selecting a patient with an unclear onset time. ${ }^{18}$ In our study, most hospitals adopting CT-based protocols as well as even one-fourth of MR-based centers were shown to adopt multiphase CTA, possibly reflecting a desire to assess collaterality or concerns about limited accessibility to post-processing programs.

\section{Current role of CTP in a real-world practice}

Evidence for the use of perfusion imaging is modest in the workflow of early time window stroke. Although CTP can provide ancillary information, such as the ability to predict early response to intravenous thrombolysis and assess the risk of hemorrhagic transformation after reperfusion therapy, this information is not directly associated with clinical decision making to proceed with EVT. ${ }^{4}$ As CTP in combination with RAPID software has become a mainstay of imaging-based eligibility criteria for EVT in a late time window, we found that the frequency of practical use of perfusion-weighted images has increased over that observed in previous nationwide surveys. ${ }^{11,19}$ However, CTP was not a preferred additional modality for patients with late time window stroke; instead, MRI was used for aiding decision-making for EVT. This phenomenon might be naturally connected with the low availability $(28 \%, 10 / 36)$ of post-processing programs for quantifying CTP data. Plausible explanations for this might include the high purchasing cost of commercial programs and the adoption of alternative eligibility criteria using MRI or angiography-based collateral imaging in each hospital.

\section{Imaging strategies in posterior circulation stroke}

Most hospitals applied reperfusion therapy for posterior circulation stroke up to 24 hours after symptom onset. Although many hospitals had adopted a wider therapeutic time window for posterior circulation stroke than for anterior circulation stroke, an analysis of the basilar artery occlusion registry showed that patients treated beyond 9 hours after symptom onset had poor clinical outcomes. ${ }^{20}$ Nevertheless, this time dependency disappeared when the results were adjusted for the baseline extent of the infarct core. Hence, many recent studies showing the clinical benefits of thrombectomy in acute basilar artery occlusion used the posterior circulation Acute Stroke Prognosis Early CT score on DWI with or without the aid of collateral scores. ${ }^{20-22}$ Mirroring the results of these studies, most hospitals used DWI when patients with posterior circulation stroke presented later than 6 hours after symptom onset. Moreover, owing to the difficulty in discriminating posterior circulation stroke from anterior circulation stroke using neurologic examination alone in clinical practice, most hospitals maintain their imaging strategies regardless of the location of intracranial LVO. 


\section{Suggestions for future revision of clinical practice guidelines of stroke}

An interesting observation from the survey was that all 11 hospitals adopting MR-based imaging work-up for AIS patients each had an individual protocol. Five centers obtained NECT with CTA prior to further acquisition of MRI, and 3 of them obtained duplicating MRA in their routine protocols. Moreover, one center obtained full sets of vascular and perfusion images using both $\mathrm{CT}$ and MRI. A fast scanning technique is applied in nearly half of them ( $n=5,45 \%)$, and the other used a conventional technique with simplified sequences to save time. These findings may be explained by a selection bias as well as the fact that the centers are likely more academically oriented, as documented by most of the sites that took the survey are university or teaching hospitals. In this, consensus on optimal MR sequences in balance with a lapse of time is needed in future revisions of clinical practice guidelines.

The most practical challenge for consecutive acquisition of CTA after NECT is the potential risk of iodinated contrast-induced nephrotoxicity. As 5 hospitals in our study routinely checked the serum creatinine level before administering contrast media for CTA, they unavoidably separated the scan sessions between CT and CTA. Guidelines have recommended that the risk of contrast-induced nephrotoxicity should never delay CTA to determine the presence or absence of LVO; hence, CTA should be immediately performed in patients who meet a predefined clinical severity threshold at a minimum and have no history of renal disease. ${ }^{23}$

Recently updated Korean clinical practice guidelines encouraged each center defining its own imaging modality rather than adhering to the eligibility criteria of 2 late time window trials allowing limited availability of RAPID. ${ }^{18}$ Consequently, the area of the greatest variability was the imaging strategy for assessing patients presenting beyond 6 hours in our survey. Especially, 10 hospitals utilizing NECT and CTA for early time window stroke all have different imaging protocols for late time window stroke. Interestingly, they commonly showed a tendency for using a variable degree of additional MRI regardless of time consumption, which is in line with the current trend for individualized treatment based on brain tissue status, rather than a time-based approach. ${ }^{24} \mathrm{Al}-$ though each hospital is expected to define its own imaging protocol to timely identify target mismatch in late time window stroke, a consensus based on updated guidelines and screening criteria of the national health insurance should be established in the future.

\section{Limitations}

This study has some limitations. Although the sample size of 36 tertiary hospitals is sufficient to obtain insights into real-world variations in imaging protocols in AIS, it is not sufficiently large to reflect the current overall situation in South Korea. In addition, because we could not obtain data on door-to-puncture times and treatment outcomes, which the participating hospitals were unwilling to disclose, we could not determine the associations between specific imaging protocols and efficiency in the workflow process or the clinical impact on treatment outcomes. Thus, we cannot recommend an optimal imaging strategy on the basis of clinical outcomes. Nevertheless, our results suggest the need for future research evaluating the impact of variations in specific imaging protocols on clinical outcomes across multiple hospitals.

\section{CONCLUSION}

This multicenter survey demonstrated a heterogeneous assortment of acute stroke imaging protocols, even at largescale and experienced stroke hospitals in South Korea. Recognizing the general trend and wide variations in imaging strategies for EVT could provide opportunities to hospitals to customize their own health care systems in a manner best suited to hospital-specific conditions. Furthermore, in the era of EVT, we have suggested areas where concrete consensus and standardization are needed for designing optimal imaging protocols in the future.

\section{SUPPLEMENTARY MATERIALS}

Supplementary materials related to this article can be found online at https://doi.org/10.5469/neuroint.2020.00199.

\section{Fund}

This study was supported by the Korean Society of Interventional Neuroradiology (KSIN) research grant 2019.

\section{Ethics Statement}

This study waived approval of the institutional review board. 


\section{Conflicts of Interest}

The authors have no conflicts to disclose.

\section{Author Contributions}

Concept and design: BK. Analysis and interpretation: BK, SY, and SCJ. Data collection: BK, SY, and SCJ. Writing the article: BK. Critical revision of the article: BK. Final approval of the article: BK. Statistical analysis: BK. Obtained funding: BK. Overall responsibility: BK.

\section{ORCID}

Byungjun Kim: https://orcid.org/0000-0001-9462-5885

Sung-Hye You: https://orcid.org/0000-0001-5005-9383

Seung Chai Jung: https://orcid.org/0000-0001-5559-7973

\section{REFERENCES}

1. Aghaebrahim A, Streib C, Rangaraju S, Kenmuir CL, Giurgiutiu DV, Horev A, et al. Streamlining door to recanalization processes in endovascular stroke therapy. J Neurointerv Surg 2017;9:340345

2. Menon BK, Sajobi TT, Zhang Y, Rempel JL, Shuaib A, Thornton J, et al. Analysis of workflow and time to treatment on thrombectomy outcome in the endovascular treatment for small core and proximal occlusion ischemic stroke (ESCAPE) randomized, controlled trial. Circulation 2016;133:2279-2286

3. Goyal M, Jadhav AP, Bonafe A, Diener H, Mendes Pereira V, Levy E, et al.; SWIFT PRIME investigators. Analysis of workflow and time to treatment and the effects on outcome in endovascular treatment of acute ischemic stroke: results from the SWIFT PRIME randomized controlled trial. Radiology 2016;279:888-897

4. Menon BK, Goyal M. Imaging paradigms in acute ischemic stroke: a pragmatic evidence-based approach. Radiology 2015;277:7-12

5. Saver JL, Goyal M, Bonafe A, Diener HC, Levy El, Pereira VM, et al.; SWIFT PRIME Investigators. Stent-retriever thrombectomy after intravenous t-PA vs. t-PA alone in stroke. $N$ Engl J Med 2015;372:2285-2295

6. Jovin TG, Chamorro A, Cobo E, de Miquel MA, Molina CA, Rovira A, et al.; REVASCAT Trial Investigators. Thrombectomy within 8 hours after symptom onset in ischemic stroke. N Engl J Med 2015;372:2296-2306

7. Goyal M, Demchuk AM, Menon BK, Eesa M, Rempel JL, Thornton J, et al.; ESCAPE Trial Investigators. Randomized assessment of rapid endovascular treatment of ischemic stroke. $N$ Engl J
Med 2015;372:1019-1030

8. Campbell BC, Mitchell PJ, Kleinig TJ, Dewey HM, Churilov L, Yassi $\mathrm{N}$, et al.; EXTEND-IA Investigators. Endovascular therapy for ischemic stroke with perfusion-imaging selection. N Engl J Med 2015;372:1009-1018

9. Berkhemer OA, Fransen PS, Beumer D, van den Berg LA, Lingsma HF, Yoo AJ, et al.; MR CLEAN Investigators. A randomized trial of intraarterial treatment for acute ischemic stroke. N Engl J Med 2015;372:11-20

10. Nogueira RG, Jadhav AP, Haussen DC, Bonafe A, Budzik RF, Bhuva P, et al.; DAWN Trial Investigators. Thrombectomy 6 to 24 hours after stroke with a mismatch between deficit and infarct. NEngl J Med 2018;378:11-21

11. Albers GW, Marks MP, Kemp S, Christensen S, Tsai JP, Ortega-Gutierrez S, et al.; DEFUSE 3 Investigators. Thrombectomy for stroke at 6 to 16 hours with selection by perfusion imaging. $N$ Engl J Med 2018;378:708-718

12. Warach SJ, Luby M, Albers GW, Bammer R, Bivard A, Campbell BC, et al.; Stroke Imaging Research (STIR) and VISTA-Imaging Investigators. Acute stroke imaging research roadmap III imaging selection and outcomes in acute stroke reperfusion clinical trials: consensus recommendations and further research priorities. Stroke 2016;47:1389-1398

13. Wintermark M, Luby M, Bornstein NM, Demchuk A, Fiehler J, Kudo K, et al. International survey of acute stroke imaging used to make revascularization treatment decisions. Int J Stroke 2015;10:759-762

14. Warach S, Gaa J, Siewert B, Wielopolski P, Edelman RR. Acute human stroke studied by whole brain echo planar diffusion-weighted magnetic resonance imaging. Ann Neurol 1995;37:231-241

15. Provost C, Soudant M, Legrand L, Ben Hassen W, Xie Y, Soize S, et al. Magnetic resonance imaging or computed tomography before treatment in acute ischemic stroke. Stroke 2019;50:659664

16. Goyal M, Menon BK, Hill MD, Demchuk A. Consistently achieving computed tomography to endovascular recanalization $<90$ minutes: solutions and innovations. Stroke 2014;45:e252-e256

17. Rocha $M$, Jovin TG. Fast versus slow progressors of infarct growth in large vessel occlusion stroke: clinical and research implications. Stroke 2017;48:2621-2627

18. Ko SB, Park HK, Kim BM, Heo JH, Rha JH, Kwon SU, et al. 2019 update of the Korean clinical practice guidelines of stroke for endovascular recanalization therapy in patients with acute ischemic stroke. Neurointervention 2019;14:71-81

19. Seo KD, Suh SH. Endovascular treatment in acute ischemic 
stroke: a nationwide survey in Korea. Neurointervention 2018;13:84-89

20. Vergouwen MD, Algra A, Pfefferkorn T, Weimar C, Rueckert CM, Thijs V, et al.; Basilar Artery International Cooperation Study (BASICS) Study Group. Time is brain(stem) in basilar artery occlusion. Stroke 2012;43:3003-3006

21. Guillaume M, Lapergue B, Gory B, Labreuche J, Consoli A, Mione G, et al.; Endovascular Treatment in Ischemic Stroke (ETIS) Investigators. Rapid successful reperfusion of basilar artery occlusion strokes with pretreatment diffusion-weighted imaging posterior-circulation aspects $<8$ is associated with good outcome. $J$ Am Heart Assoc 2019;8:e010962

22. Goyal N, Tsivgoulis G, Nickele C, Doss VT, Hoit D, Alexandrov AV, et al. Posterior circulation CT angiography collaterals predict outcome of endovascular acute ischemic stroke therapy for basilar artery occlusion. J Neurointerv Surg 2016;8:783-786

23. McTaggart RA, Ansari SA, Goyal M, Abruzzo TA, Albani B, Arthur AJ, et al.; Standards and Guidelines Committee of the Society of Neurolnterventional Surgery (SNIS). Initial hospital management of patients with emergent large vessel occlusion (ELVO): report of the standards and guidelines committee of the Society of Neurolnterventional Surgery. J Neurointerv Surg 2017;9:316-323

24. Demeestere J, Wouters A, Christensen S, Lemmens R, Lansberg $M G$. Review of perfusion imaging in acute ischemic stroke: from time to tissue. Stroke 2020;51:1017-1024 